\title{
Corrigendum: Host DNA released by NETosis promotes rhinovirus-induced type-2 allergic asthma exacerbation
}

Marie Toussaint, David J Jackson, Dawid Swieboda, Anabel Guedán, Theodora-Dorita Tsourouktsoglou, Yee Man Ching, Coraline Radermecker, Heidi Makrinioti, Julia Aniscenko, Michael R Edwards, Roberto Solari, Frédéric Farnir, Venizelos Papayannopoulos, Fabrice Bureau, Thomas Marichal \& Sebastian L Johnston

Nat. Med. 23, 681-691 (2017); published online 1 May 2017; corrected after print 12 July 2017

In the version of this article initially published, Dr. Nathan W Bartlett was inadvertently omitted from the author list and the Contributions section. The errors have been corrected in the HTML and PDF versions of the article.

\section{Corrigendum: The cold-induced lipokine 12,13-diHOME promotes fatty acid transport into brown adipose tissue}

Matthew D Lynes, Luiz O Leiria, Morten Lundh, Alexander Bartelt, Farnaz Shamsi, Tian Lian Huang, Hirokazu Takahashi, Michael F Hirshman, Christian Schlein, Alexandra Lee, Lisa A Baer, Francis J May, Fei Gao, Niven R Narain, Emily Y Chen, Michael A Kiebish, Aaron M Cypess, Matthias Blüher, Laurie J Goodyear, Gökhan S Hotamisligil, Kristin I Stanford \& Yu-Hua Tseng Nat. Med. 23, 631-637 (2017); published online 27 March 2017; corrected after print 23 August 2017

In the phrase, "Here we show that the lipid 12,13-dihydroxy-9Z-octadecenoic acid (12,13-diHOME) is a stimulator of BAT activity, and that its levels are negatively correlated with body-mass index and insulin sensitivity," located in the abstract, the word "resistance" should take the place of the word "sensitivity".

Also, the authors have clarified in more detail how the FATP1 oligomer density was quantitated in Figure 4f. This information can be found in the "Membrane Fractionation" section of the Online Methods: "To quantify FATP1 in scanned immunoblots, regions of interest of identical size were drawn in each lane at the same molecular weight, and integrated pixel density was measured using ImageJ software. For each independent experimental replicate, the integrated pixel density for each lane was expressed normalized to the control lane, or in the case of the experimental replicate with two control lanes, the integrated pixel density for each lane was expressed normalized to the average of both control lanes. The data are expressed as the average normalized value for each lane, with the error bars representing s.e.m."

Corrigendum: Targeting cellular senescence prevents age-related bone loss in mice

Joshua N Farr, Ming Xu, Megan M Weivoda, David G Monroe, Daniel G Fraser, Jennifer L Onken, Brittany A Negley, Jad G Sfeir, Mikolaj B Ogrodnik, Christine M Hachfeld, Nathan K LeBrasseur, Matthew T Drake, Robert J Pignolo, Tamar Pirtskhalava, Tamara Tchkonia, Merry Jo Oursler, James L Kirkland \& Sundeep Khosla

Nat. Med. 23, 1072-1079 (2017); published online 21 August 2017; corrected after print 11 October 2017

In the version of this article initially published, the representative images in Fig. $2 \mathrm{n}$ were inadvertently duplicated in Fig. $4 \mathrm{~h}$. A correct version of the images for Fig. $4 \mathrm{~h}$ has been supplied. The authors wish to point out that this error did not affect the other data reported in the paper or the conclusions drawn. The error has been corrected in the HTML and PDF versions of the article.

\section{Corrigendum: Genome-wide CRISPR screens reveal a Wnt-FZD5 signaling circuit as a druggable vulnerability of RNF43-mutant pancreatic tumors}

Zachary Steinhart, Zvezdan Pavlovic, Megha Chandrashekhar, Traver Hart, Xiaowei Wang, Xiaoyu Zhang, Mélanie Robitaille, Kevin R Brown, Sridevi Jaksani, René Overmeer, Sylvia F Boj, Jarrett Adams, James Pan, Hans Clevers, Sachdev Sidhu, Jason Moffat \& Stéphane Angers

Nat. Med. 23, 60-68 (2017); published online 21 November 2016; corrected after print 20 September 2017

In the version of this article initially published, duplicate panels in Figure 5e were incorrectly used in the assembly of the figure, for which all original panels are presented in Supplementary Figure 10. The errors in Figure 5e have been corrected in the HTML and PDF versions of the article. 